\title{
Mobilization of metal(oid) oxyanions through circumneutral mine waste-rock drainage
}

Bas Vriens ${ }^{\dagger}{ }^{*}$, Elliott K. Skierszkan ${ }^{\dagger}$, Melanie St-Arnault ${ }^{\ddagger}$, Kristin Salzsauler§, Celedonio Aranda", K. Ulrich Mayer ${ }^{\dagger}$, Roger D. Beckie ${ }^{\dagger}$

† Department of Earth, Ocean and Atmospheric Sciences, University of British Columbia, 2020-2207 Main Mall, Vancouver BC, V6T 1Z4, Canada

‡ Norman B. Keevil Institute of Mining Engineering, University of British Columbia, 2329 West Mall, Vancouver, BC, V6T 1Z4, Canada

§ Golder Associates, Suite 200 - 2920 Virtual Way, Vancouver, BC, V5M 0C4, Canada

" Compañia Antamina Minera S.A., Av. El Derby No. 055, Santiago de Surco, 15023 Lima, Peru

* Corresponding author e-mail: bvriens@eoas.ubc.ca 


\title{
Supporting Information
}

\author{
Supporting Methods \\ Supporting Tables S1-S6 \\ Supporting Figures S1-S10
}




\section{Supporting Methods}

\section{Chemical equilibrium modeling}

Chemical equilibrium and surface complexation modeling was performed in PHREEQC. Plots of the aqueous equilibrium speciation of $\mathrm{As}, \mathrm{Sb}$, Mo and $\mathrm{Se}$ were constructed using the MINTEQ.v4 database at $25^{\circ} \mathrm{C}$, at $1<\mathrm{pH}<11$ and under oxic conditions ( $\mathrm{pe}=5$ : sporadic measurements of $\mathrm{O}_{2}$-saturation in the drainage indicated near-complete saturation (data not shown)). No polyoxometalates (e.g., $\mathrm{H}_{2} \mathrm{Mo}_{7} \mathrm{O}_{24}{ }^{6-}$ ) were considered for the aqueous molybdate speciation. The surface speciation of hydrous ferric oxides (HFO) under variable $\mathrm{pH}$ was investigated using the abovementioned database and the Dzombak and Morel ${ }^{1}$ diffuse double layer model (no explicit layer defined). For illustrative purposes, a hypothetical phase (1 $\mathrm{g}$ of HFO with a surface area of $600 \mathrm{~m}^{2} / \mathrm{g}$ and $0.5 \mathrm{mmol}$ of strong- and $20 \mathrm{mmol}$ of weak sorption sites) was equilibrated with either pure solutions of $5 \mathrm{mM}$ of each of the individual elements (hydrolysis only; Figure 4) or with more complex solutions that were based on recorded averaged drainage chemistries (Figure S10). For the latter simulations, the used average drainage chemistries across the $\mathrm{pH}$ range $2-10$ are given in Table S4. For all simulations, the $\mathrm{pH}$ was fixed, charge balance was achieved through $\mathrm{Na}$ and the redox equilibrium was adjusted to pe.

[1]: Dzombak, D.A.; Morel, F. Surface Complexation Modeling: Hydrous Ferric Oxide, John Wiley \& Sons, New York, Mar. 16, 1990, 393 pages. 
Table S1. Bulk geochemical characteristics of the unweathered waste rock used in the field barrel kinetic experiments.

\begin{tabular}{|c|c|c|c|c|c|c|c|c|c|c|}
\hline $\begin{array}{l}\text { Field } \\
\text { barrel }\end{array}$ & $\begin{array}{l}\text { Weight } \\
{[\mathrm{kg}]}\end{array}$ & $\begin{array}{l}\mathrm{NPR}^{* *} \\
{[-]}\end{array}$ & $\begin{array}{l}\text { Sulfide } \\
{[\%]}\end{array}$ & $\begin{array}{l}\text { TIC } \\
{[\%]}\end{array}$ & $\begin{array}{l}\mathrm{Fe} \\
{[\%]}\end{array}$ & $\begin{array}{l}\mathrm{Ca} \\
{[\%]}\end{array}$ & $\begin{array}{l}\text { As } \\
{[\mathrm{mg} / \mathrm{kg}]}\end{array}$ & $\begin{array}{l}\mathrm{Mo} \\
{[\mathrm{mg} / \mathrm{kg}]}\end{array}$ & $\begin{array}{l}\mathrm{Sb} \\
{[\mathrm{mg} / \mathrm{kg}]}\end{array}$ & $\begin{array}{l}\mathrm{Se} \\
{[\mathrm{mg} / \mathrm{kg}]}\end{array}$ \\
\hline \multicolumn{11}{|c|}{ Intrusive waste rock } \\
\hline 11 & 350 & 1.6 & 0.9 & 0.5 & 2 & 2 & 98 & 250 & 5 & 2 \\
\hline 12 & 300 & 1.1 & 0.6 & 0.1 & 3 & 3 & 18 & 368 & 6 & $<2$ \\
\hline 13 & 325 & 2.6 & 0.7 & 0.7 & 3 & 6 & 53 & 180 & 8 & $<2$ \\
\hline 14 & 350 & 1.5 & 3.7 & 0.2 & 2 & 1 & 167 & 265 & 8 & 3 \\
\hline 15 & 350 & 1.9 & 1.3 & 0.1 & 1 & 1 & 54 & 429 & 5 & 2 \\
\hline 16 & 350 & 0.1 & 4.2 & 0.1 & 10 & 1 & 21 & 329 & 4 & 7 \\
\hline 17 & 325 & 0.4 & 0.6 & 0.1 & 1 & 1 & 70 & 393 & 7 & 2 \\
\hline 18 & 350 & 0.5 & 1.7 & 0.1 & 3 & 2 & 101 & 203 & $<2$ & $<2$ \\
\hline 19 & 350 & 0.1 & 5.2 & 0.1 & 11 & 2 & 228 & 282 & 11 & 5 \\
\hline 110 & 350 & 1.2 & 2.1 & 0.1 & 15 & 10 & 73 & 130 & 8 & 4 \\
\hline $\mid 11$ & 350 & 0.4 & 1.4 & 0.1 & 3 & 2 & 102 & 132 & $<2$ & $<2$ \\
\hline \multirow[t]{4}{*}{ I12 } & 350 & 1.7 & 1.5 & 0.1 & 8 & 1 & 122 & 100 & $<2$ & $<2$ \\
\hline & Average & 1.1 & 2.0 & 0.2 & 5 & 3 & 92 & 255 & $<5$ & $<3$ \\
\hline & STDEV & 0.8 & 1.5 & 0.2 & 4 & 3 & 57 & 105 & $<3$ & $<2$ \\
\hline & \multicolumn{10}{|c|}{ Skarn waste rock } \\
\hline S1 & 350 & 3 & 0.4 & 0.4 & 3 & 7 & 13 & 1,798 & $<2$ & $<2$ \\
\hline S2 & 350 & 4 & 0.4 & 0.6 & 2 & 4 & 20 & 588 & $<2$ & $<2$ \\
\hline S3 & 350 & 4 & 4.3 & 1.9 & 7 & 15 & 31 & 948 & 6 & 24 \\
\hline S4 & 350 & 4 & 0.9 & 1.3 & 3 & 12 & 62 & 78 & 13 & 7 \\
\hline S5 & 350 & 5 & 4.5 & 2 & 4 & 19 & 41 & 43 & 4 & 15 \\
\hline S6 & 325 & 6 & 0.1 & 3 & 4 & 20 & 47 & 53 & 4 & 4 \\
\hline S7 & 350 & 2 & 2.8 & 1.0 & 13 & 19 & 67 & 306 & 12 & 16 \\
\hline S8 & 350 & 1 & 9.6 & 0.3 & 12 & 16 & 36 & 132 & 6 & 21 \\
\hline S9 & 350 & 1 & 6.2 & 0.8 & 13 & 17 & 77 & 68 & 29 & 20 \\
\hline S10 & 325 & 8 & 2.7 & 3.1 & 6 & 22 & 1,050 & 156 & 137 & 13 \\
\hline \multirow[t]{3}{*}{ S11 } & 350 & 4 & 1.7 & 0.7 & 9 & 21 & 46 & 116 & 4 & 5 \\
\hline & Average & 4 & 3 & 1.4 & 7 & 16 & 135 & 390 & $<20$ & $<12$ \\
\hline & STDEV & 2 & 3 & 0.9 & 4 & 6 & 290 & 520 & $<38$ & $<8$ \\
\hline
\end{tabular}

${ }^{* *} N P R$ : neutralization potential ratio $=$ acid neutralizing potential $(N P) /$ acid production potential $(A P)$ 
Table S1, cont.

\begin{tabular}{lllllllllll}
\hline $\begin{array}{l}\text { Field } \\
\text { barrel }\end{array}$ & $\begin{array}{l}\text { Weight } \\
{[\mathrm{kg}]}\end{array}$ & $\begin{array}{l}\text { NPR } \\
{[-]^{* *}}\end{array}$ & $\begin{array}{l}\text { Sulfide } \\
{[\%]}\end{array}$ & $\begin{array}{l}\mathrm{TIC}] \\
{[\%]}\end{array}$ & $\begin{array}{l}\mathrm{Fe} \\
{[\%]}\end{array}$ & $\begin{array}{l}\mathrm{Ca} \\
{[\%]^{*}}\end{array}$ & $\begin{array}{l}\mathrm{As} \\
{[\mathrm{mg} / \mathrm{kg}]}\end{array}$ & $\begin{array}{l}\mathrm{Mo} \\
{[\mathrm{mg} / \mathrm{kg}]}\end{array}$ & $\begin{array}{l}\mathrm{Sb} \\
{[\mathrm{mg} / \mathrm{kg}]}\end{array}$ & $\begin{array}{l}\mathrm{Se} \\
{[\mathrm{mg} / \mathrm{kg}]}\end{array}$ \\
\hline $\mathrm{H} 1$ & 300 & 14 & 0.9 & 5 & 2 & $>15$ & 36 & $<2$ & $<2$ & $<2$ \\
$\mathrm{H} 2$ & 350 & 21 & 0.9 & 7 & 2 & $>15$ & 31 & 4 & 4 & 3 \\
$\mathrm{H} 3$ & 325 & 28 & 0.2 & 2 & 3 & 9 & 190 & $<2$ & 14 & $<2$ \\
$\mathrm{H} 4$ & 325 & 23 & 0.7 & 6 & 2 & $>15$ & 25 & 44 & $<2$ & $<2$ \\
$\mathrm{H} 5$ & 350 & 29 & 0.6 & 11 & 1 & 36 & 23 & 6 & 6 & 2 \\
$\mathrm{H} 6$ & 350 & 12 & 2.0 & 3 & 2 & 26 & 36 & 100 & $<2$ & $<2$ \\
$\mathrm{H} 7$ & 350 & 41 & 0.2 & 3 & 1 & 29 & 46 & 3 & $<2$ & $<2$ \\
$\mathrm{H} 8$ & 350 & 41 & 1.2 & 10 & 1 & 24 & 130 & 10 & $<2$ & $<2$ \\
$\mathrm{H} 9$ & 350 & 51 & 0.5 & 9 & 1 & 30 & 84 & 5 & 3 & $<2$ \\
$\mathrm{H} 10$ & 350 & 39 & 2.1 & 3 & 3 & 34 & 102 & $<2$ & 5 & $<2$ \\
$\mathrm{H} 11$ & 350 & 9 & 1.3 & 2 & 2 & 29 & 127 & $<2$ & 14 & $<2$ \\
$\mathrm{H} 12$ & 350 & 41 & 0.6 & 4 & 2 & 27 & 251 & $<2$ & $<2$ & $<2$ \\
$\mathrm{H} 13$ & 350 & 39 & 0.4 & 2 & 2 & 29 & 116 & $<2$ & $<2$ & $<2$ \\
$\mathrm{H} 14$ & 350 & 40 & 1.3 & 10 & 1 & 23 & 74 & 289 & 13 & $<2$ \\
& Average & 31 & 0.9 & 6 & 1.8 & $>24$ & 91 & $<33$ & $<5$ & $<1.2$ \\
& STDEV & 13 & 0.6 & 3 & 0.7 & $>8$ & 65 & $<76$ & $<5$ & $<0.6$ \\
\hline
\end{tabular}

\begin{tabular}{lllllllllll}
\hline \multicolumn{1}{c}{ Marble waste rock } \\
\hline M1 & 350 & 708 & 0.1 & 11 & 1 & $>15$ & 68 & 18 & 7 & $<2$ \\
M2 & 325 & 45 & 0.6 & 11 & 1 & $>15$ & 35 & 10 & $<2$ & $<2$ \\
M3 & 350 & 31 & 0.6 & 9 & 2 & $>15$ & 15 & 4 & $<2$ & $<2$ \\
M4 & 350 & 39 & 0.4 & 7 & 1 & 26 & 92 & $<2$ & $<2$ & $<2$ \\
M5 & 350 & 164 & 0.5 & 9 & 1 & 32 & 194 & 11 & 13 & 2 \\
M6 & 350 & 221 & 0.1 & 9 & 1 & 33 & 74 & 2 & 11 & 2 \\
M7 & 350 & 75 & 0.9 & 7 & 1 & 29 & 73 & 104 & 20 & 3 \\
M8 & 350 & 35 & 0.4 & 8 & 2 & 33 & 34 & 63 & 4 & 6 \\
M9 & 350 & 29 & 0.6 & 6 & 1 & 28 & 102 & 3 & $<2$ & $<2$ \\
M10 & 350 & 30 & 2.5 & 5 & 2 & 25 & 53 & 5 & $<2$ & $<2$ \\
M11 & 350 & 112 & 0.1 & 10 & 2 & 36 & 16 & 10 & $<2$ & $<2$ \\
M12 & 325 & 31 & 0.8 & 11 & 1 & 37 & 16 & $<2$ & 3 & $<2$ \\
& Average & 127 & 0.6 & 9 & 1.3 & $>27$ & 65 & $<19$ & $<5$ & $<2$ \\
& STDEV & 185 & 0.6 & 2 & 0.5 & $>8$ & 51 & $<30$ & $<6$ & $<1$ \\
\hline
\end{tabular}

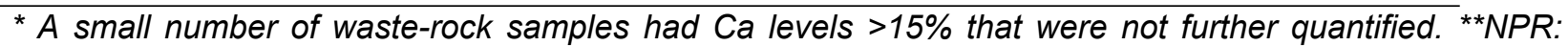
neutralization potential ratio $=$ acid neutralizing potential (NP) / acid production potential (AP) 
Table S2. Bulk mineralogy (XRD) of the unweathered waste rock used in the field barrel kinetic experiments.

\begin{tabular}{llllllllll}
\hline & $\mathbf{I 1}$ & $\mathbf{I 3}$ & $\mathbf{I 4}$ & $\mathbf{I 5}$ & $\mathbf{I 6}$ & $\mathbf{I 7}$ & $\mathbf{I 9}$ & $\mathbf{I 1 0}$ & $\mathbf{I 1 2}$ \\
\hline Actinolite & & & & & 1.4 & & 1.0 & & \\
Albite & & & 2.8 & 2.8 & 1.5 & 6.7 & 1.0 & 1.0 & 1.2 \\
Biotite & 4.0 & 3.3 & 3.3 & 2.8 & 2.1 & 3.0 & 2.0 & 2.3 & 3.1 \\
Calcite & 4.1 & 7.1 & & & & & & & \\
Chalcopyrite & 0.7 & 0.6 & 1.2 & 0.8 & 4.1 & 1.0 & 2.4 & 0.6 & 0.7 \\
Chlorites & 0.2 & 0.3 & & & & & & & \\
Feldspar & 37.7 & 34.3 & & & & & & & \\
Garnet & 1.0 & 8.1 & 1.0 & & 22.1 & 2.1 & 8.5 & 7.9 & 1.5 \\
Iron Oxides & 0.6 & 0.4 & & 0.2 & 3.4 & & 0.7 & & \\
K-feldspar & & & 41.9 & 42.3 & 22.4 & 40.1 & 34.2 & 34.0 & 44.6 \\
Melanterite & & & & & & & 0.2 & & \\
Molybdenite & 0.1 & & 0.0 & 0.1 & 0.0 & 0.0 & 0.0 & & \\
Muscovite & & & 2.3 & 2.2 & & & 0.9 & & \\
Oligoclase & & & 8.4 & 6.4 & 6.1 & 8.7 & 6.2 & 7.0 & 8.4 \\
Others & 4.7 & 0.9 & & & & & & & \\
Pyrite & 0.9 & 2.3 & 1.5 & 1.5 & 5.0 & 1.7 & 4.7 & 0.5 & 0.7 \\
Pyrrhotite & 0.7 & 0.1 & & & & & & 1.0 & \\
Pyroxene & 1.0 & 6.2 & & 1.9 & & & 2.7 & & \\
Quartz & 44.0 & 34.9 & 37.3 & 38.8 & 31.9 & 35.7 & 35.2 & 45.7 & 39.7 \\
Siderite & & & 0.2 & 0.2 & & 0.5 & & & 0.1 \\
Sphalerite & & 0.1 & & & & & & & \\
Titanium & 0.3 & 0.7 & & & & & & & \\
\hline Total & $\mathbf{1 0 0 . 0}$ & $\mathbf{9 9 . 3}$ & $\mathbf{9 9 . 9}$ & $\mathbf{1 0 0 . 0}$ & $\mathbf{1 0 0 . 0}$ & $\mathbf{9 9 . 4}$ & $\mathbf{9 9 . 9}$ & $\mathbf{1 0 0 . 0}$ & $\mathbf{1 0 0 . 0}$
\end{tabular}


Table S2, cont.

\begin{tabular}{|c|c|c|c|c|c|c|}
\hline & S4 & S5 & S7 & S8 & S10 & S11 \\
\hline Apatite & 0.4 & & & & & \\
\hline Biotite & 3.5 & 0.5 & & & & \\
\hline Calcite & 9.6 & 10.5 & 4.6 & 2.8 & 30.7 & 7.8 \\
\hline Chalcopyrite & 0.7 & 0.8 & & & & \\
\hline Chlorites & 0.5 & & 0.5 & & 3.1 & \\
\hline Diopside & & 15.4 & 10.8 & 13.1 & 10.3 & 11.8 \\
\hline Feldspar & 18.0 & & 2.9 & 0.7 & & 0.9 \\
\hline Galena & 0.1 & 0.1 & & & & \\
\hline Garnet & 27.3 & & 52.1 & 59.8 & 36.6 & 59.1 \\
\hline Iron Oxides & 0.6 & 1.2 & & & & \\
\hline K-feldspar & & 17.2 & 11.2 & 3.6 & & 1.5 \\
\hline Lazurite & & 33.0 & & & & \\
\hline Others & 0.6 & 2.1 & 0.1 & & & \\
\hline Phlogopite & & 5.2 & 0.6 & & & \\
\hline Pyrite & 1.3 & 3.4 & 1.3 & 9.0 & 0.1 & 0.7 \\
\hline Pyroaurite & & 0.5 & & & 0.6 & \\
\hline Pyroxene & 9.0 & & & & & \\
\hline Pyrrhotite & 1.2 & 0.0 & 0.7 & 0.2 & 0.6 & 0.3 \\
\hline Quartz & 14.6 & 9.8 & 3.2 & 5.4 & 3.2 & 0.9 \\
\hline Siderite & & 0.0 & & & & 0.2 \\
\hline Sphalerite & 1.0 & 0.2 & 0.8 & 0.4 & 1.9 & 0.8 \\
\hline Titanium & 0.4 & & & & & \\
\hline Vesuvianite & 11.2 & & 7.5 & 3.0 & 7.6 & 14.7 \\
\hline Wollastonite & & & 3.9 & 1.4 & 5.3 & 1.0 \\
\hline Total & 100.0 & 99.8 & 100.0 & 99.5 & 100.0 & 99.6 \\
\hline
\end{tabular}


Table S2, cont.

\begin{tabular}{|c|c|c|c|c|c|c|c|c|}
\hline & $\mathrm{H} 2$ & H5 & H6 & H8 & H9 & H10 & H11 & H14 \\
\hline Actinolite & & & 2.0 & & 3.5 & 0.2 & 0.4 & 3.2 \\
\hline Albite & & & 2.4 & 4.3 & 4.2 & 11.1 & 5.7 & 1.1 \\
\hline Anorthite & 10.2 & & 9.3 & 5.6 & & & & 0.2 \\
\hline Apatite & 0.4 & & & & & & & \\
\hline Biotite & 2.1 & 1.3 & & & & & & \\
\hline Calcite & 61.7 & 90.6 & 61.4 & 64.8 & 78.9 & 59.9 & 82.2 & 86.6 \\
\hline Chalcopyrite & 0.1 & 0.1 & & & & & & \\
\hline Chlorites & 0.2 & & 2.0 & 2.7 & 0.6 & 3.0 & & 0.4 \\
\hline Diopside & & 1.4 & 1.4 & 0.7 & 2.5 & & 0.7 & 3.2 \\
\hline Feldspar & 10.3 & & & & & & & \\
\hline Galena & 0.4 & 0.0 & & & & & & \\
\hline Garnet & 6.6 & & & & & & & \\
\hline K-feldspar & & 2.5 & 4.8 & 3.5 & 3.2 & 4.7 & & 1.7 \\
\hline Magnetite & & 0.1 & & & & & & \\
\hline Melanterite & & 0.0 & & & 0.2 & & & \\
\hline Muscovite & & 0.1 & 1.2 & 1.5 & & 3.8 & & \\
\hline Others & 2.9 & 1.5 & & & & & & \\
\hline Phlogopite & & 0.4 & 4.7 & 4.6 & 1.8 & 4.6 & 4.4 & 0.2 \\
\hline Pyrite & 0.9 & 0.5 & 0.3 & & & 0.1 & 0.5 & 1.2 \\
\hline Pyroaurite & & 0.1 & & & & & & \\
\hline Pyrrhotite & 2.0 & 0.0 & 1.8 & 1.2 & 1.6 & 2.1 & 0.8 & 0.9 \\
\hline Quartz & 0.8 & 1.3 & 8.7 & 11.0 & 3.4 & 10.3 & 5.0 & 1.2 \\
\hline Sphalerite & 0.2 & 0.0 & & & & & & \\
\hline Total & 98.7 & 99.9 & 100.0 & 100.0 & 100.0 & 100.0 & 99.6 & 100.0 \\
\hline
\end{tabular}


Table S2, cont.

\begin{tabular}{llllllllll}
\hline & M1 & M2 & M4 & M5 & M7 & M8 & M9 & M11 & M12 \\
\hline Actinolite & & & & & & & 1.1 & 3.2 & 2.6 \\
Albite & & & & & & & 3.1 & 1.1 & 0.0 \\
Anorthite & & & & & & & 9.1 & 0.2 & 0.7 \\
Apatite & 0.4 & 0.3 & 0.6 & & & & & & \\
Biotite & 0.1 & 1.6 & 2.1 & 1.6 & 1.5 & 1.4 & & & \\
Calcite & 84.8 & 90.2 & 55.5 & 55.2 & 47.9 & 65.5 & 57.6 & 86.6 & 91.9 \\
Chalcopyrite & 0.2 & 0.4 & & 0.1 & 0.7 & 0.4 & & & \\
Chlorites & 0.1 & & 0.1 & & & & 1.6 & 0.4 & 0.3 \\
Galena & 0.1 & 0.1 & 0.1 & 0.0 & 0.1 & 0.1 & & & \\
Garnet & 5.6 & 0.7 & 10.2 & & & & & & \\
Iron Oxides & 0.2 & 0.1 & & 1.1 & 0.4 & 0.3 & & & \\
K-feldspar & 2.1 & & 5.0 & 5.7 & 7.3 & 2.5 & 7.6 & 1.7 & 0.9 \\
Lazurite & & & & 6.4 & 17.4 & 13.3 & & & \\
Others & 0.2 & 0.4 & 0.8 & 3.5 & 0.8 & 0.9 & & & 0.5 \\
Phlogopite & & & & 4.1 & 3.1 & 1.3 & 4.0 & 0.2 & 0.8 \\
Plagioclase & & & & 5.2 & 3.1 & 3.8 & & & \\
Pyrite & 0.1 & 0.1 & 0.2 & 0.2 & 0.7 & 1.5 & 1.1 & 1.2 & \\
Pyroxene & 5.1 & 1.2 & 8.6 & 7.9 & 7.6 & 5.5 & 3.9 & 3.2 & 1.1 \\
Pyrrhotite & & 0.1 & 0.7 & 0.1 & 0.2 & 0.3 & 1.5 & 0.9 & 0.8 \\
Quartz & 0.7 & 3.6 & 1.1 & 8.3 & 8.7 & 2.9 & 9.3 & 1.2 & 0.4 \\
Sphalerite & & 0.5 & & 0.1 & 0.3 & 0.2 & & & \\
Titanium & 0.1 & & 0.7 & & & & & & \\
Vesuvianite & & & 13.8 & & & & & & \\
\hline Total & $\mathbf{9 9 . 7}$ & $\mathbf{9 9 . 2}$ & $\mathbf{9 9 . 4}$ & $\mathbf{9 9 . 4}$ & $\mathbf{9 9 . 7}$ & $\mathbf{9 9 . 8}$ & $\mathbf{1 0 0 . 0}$ & $\mathbf{1 0 0 . 0}$ & $\mathbf{1 0 0 . 0}$
\end{tabular}


Table S3. Multivariate regression analysis between elemental drainage concentrations recorded in the field barrel experiments. For this analysis, recorded elemental drainage concentrations from all field barrels $(n=5,518$; measurements below their respective detection limit [ $n=980]$ set to half that limit) were log-normalized. Missing values in the covariance matrix were estimated using the unstructured maximum likelihood. Numbers indicate the correlation coefficients $r$ between log-normalized elemental concentrations. A selection of correlation plots is given in Figure S4.

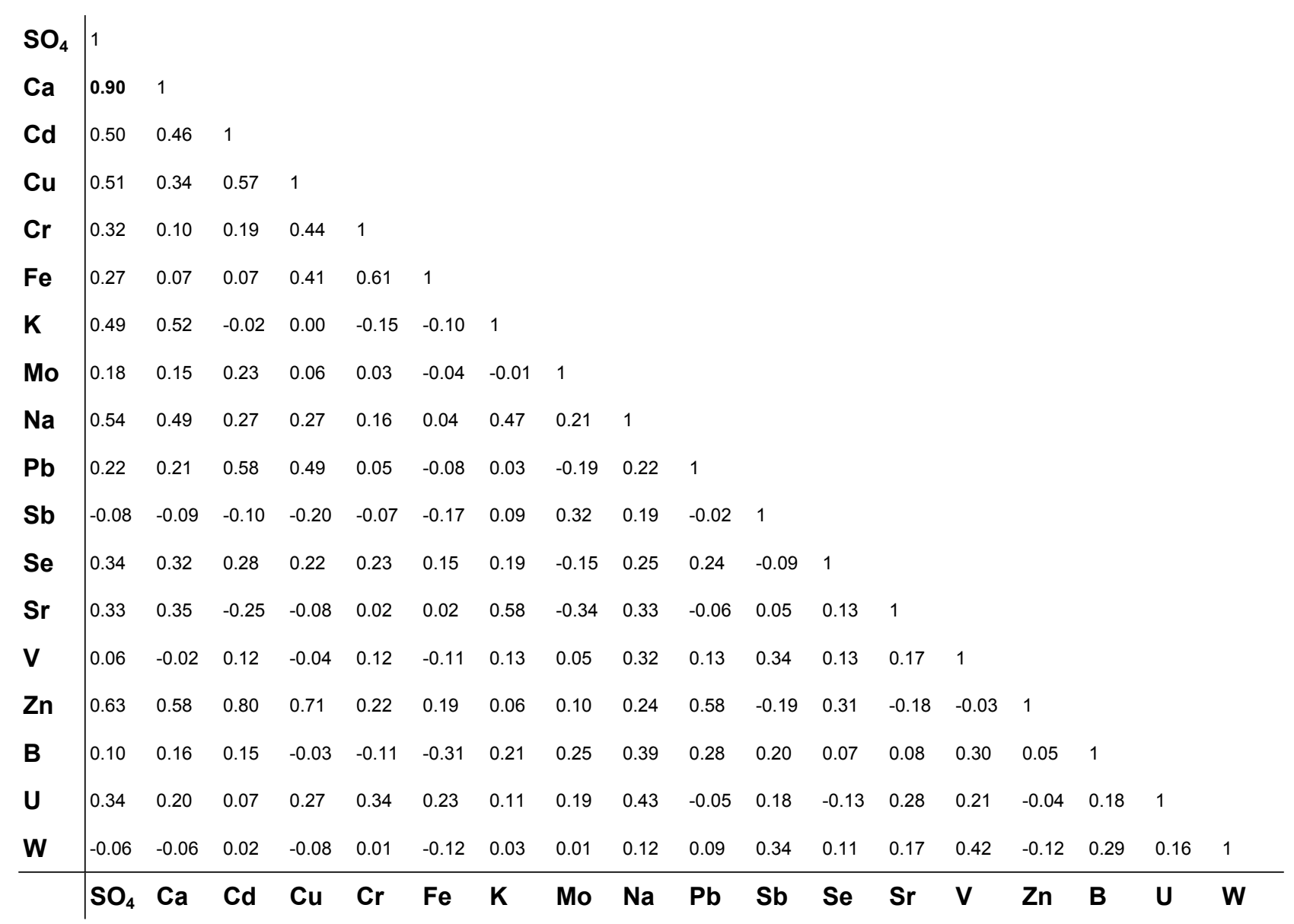


Table S4. Average drainage chemistries recorded in the field barrel experiments that were used for the simulation of $\mathrm{As}, \mathrm{Sb}, \mathrm{Mo}$ and Se sorption onto HFO. All surface complexation simulations were performed at $10^{\circ} \mathrm{C}$ by equilibrating below-mentioned solutions with $1 \mathrm{~g}$ of HFO with a surface area of $600 \mathrm{~m}^{2} / \mathrm{g}$ and $0.5 \mathrm{mmol}$ of strong- and $20 \mathrm{mmol}$ of weak sorption sites.

\begin{tabular}{|c|c|c|c|c|c|c|c|c|c|c|}
\hline Parameter & Averag & value $\mathrm{i}$ & draina & ge $[\mathrm{mg}$ & - excep & $\mathrm{pH} \&$ & & & & \\
\hline $\mathrm{pH}$ & $1^{*}$ & 2 & 3 & 4 & 5 & 6 & 7 & 8 & 9 & $10^{*}$ \\
\hline pe & 5 & 5 & 5 & 5 & 5 & 5 & 5 & 5 & 5 & 5 \\
\hline$[T I C]^{\star *}$ & 0 & 0 & 0 & 0 & 0.01 & 1.5 & 1.5 & 10 & 50 & 50 \\
\hline$[S]_{\text {total }}$ & 10,000 & 7,500 & 6,000 & 5,000 & 5,000 & 5,000 & 5,000 & 5,000 & 1,000 & 1,000 \\
\hline$[\mathrm{Fe}]$ & 1 & 1 & 1 & 0.1 & 0 & 0 & 0 & 0 & 0 & 0 \\
\hline [Ca] & 150 & 150 & 150 & 150 & 150 & 250 & 500 & 600 & 800 & 1,000 \\
\hline$[\mathrm{Na}],[\mathrm{K}]$ & \multicolumn{10}{|c|}{5} \\
\hline [Si] & \multicolumn{10}{|c|}{10} \\
\hline$[\mathrm{Mn}],[\mathrm{Mg}],[\mathrm{Sr}]$ & \multicolumn{10}{|c|}{$<1$} \\
\hline $\begin{array}{l}\text { [As] or [Mo] or } \\
{[\mathrm{Sb}] \text { or }[\mathrm{Se}]}\end{array}$ & \multicolumn{10}{|c|}{$0.05-50$} \\
\hline
\end{tabular}

* These $\mathrm{pH}$ ranges were not observed in the field experiments but their composition estimated by extrapolation for illustrative purposes.

** Drainage alkalinity was assumed to constitute total inorganic carbon [TIC] only. 
Table S5. Analytical detection limits for aqueous drainage chemistry measurements.

Ranges indicate improvement during the $>10 \mathrm{yr}$ monitoring program.

\begin{tabular}{|l|l|}
\hline Element & Detection limit [mg/L] \\
\hline As & 0.001 \\
\hline $\mathrm{B}$ & $0.025-0.01$ \\
\hline $\mathrm{Cd}$ & 0.003 \\
\hline $\mathrm{Co}$ & 0.003 \\
\hline $\mathrm{Cr}$ & 0.002 \\
\hline $\mathrm{Fe}$ & 0.001 \\
\hline $\mathrm{Mo}$ & $0.01-0.001$ \\
\hline $\mathrm{P}$ & $0.3-0.02$ \\
\hline $\mathrm{Pb}$ & 0.0004 \\
\hline $\mathrm{Sb}$ & $0.01-0.0008$ \\
\hline $\mathrm{Se}$ & $0.01-0.002$ \\
\hline $\mathrm{Si}$ & 0.05 \\
\hline $\mathrm{TI}$ & 0.003 \\
\hline $\mathrm{V}$ & $0.007-0.002$ \\
\hline $\mathrm{W}$ & 0.0002 \\
\hline
\end{tabular}


Table S6. Elemental concentration ratios of $\mathrm{As}, \mathrm{Mo}, \mathrm{Se}$ and $\mathrm{Sb}$ in the average drainage (aqueous phase) over the waste rock (solid phase) for all field barrels.

\begin{tabular}{|c|c|c|c|c|c|c|c|c|c|c|c|c|c|c|c|c|}
\hline \multirow{3}{*}{$\begin{array}{l}\text { Field } \\
\text { barrel }\end{array}$} & \multicolumn{4}{|c|}{$\begin{array}{l}\text { Solid-phase concentration } \\
\text { in waste rock [mg/kg] }\end{array}$} & \multicolumn{8}{|c|}{ Aqueous concentration in drainage $[\mathrm{mg} / \mathrm{L}]$} & \multicolumn{4}{|c|}{$\begin{array}{l}\text { LOG Elemental ratios } \\
\text { drainage/solid-phase } \\
{[\mathrm{kg} / \mathrm{L}]}\end{array}$} \\
\hline & \multirow[b]{2}{*}{ As } & \multirow[b]{2}{*}{ Mo } & \multirow[b]{2}{*}{$\mathrm{Sb}$} & \multirow[b]{2}{*}{ Se } & As & & Mo & & $\mathrm{Sb}$ & & $\mathrm{Se}$ & & & & & \\
\hline & & & & & mean & stdev & mean & stdev & mean & stdev & mean & stdev & As & Mo & $\mathrm{Sb}$ & $\mathrm{Se}$ \\
\hline 11 & 98 & 250 & 5 & 2 & 0.1 & 0.07 & 5.2 & 4.4 & 0.07 & 0.04 & 0.003 & 0.002 & -3 & -2 & -2 & -3 \\
\hline 12 & 18 & 368 & 6 & $<2$ & 0.02 & 0.007 & 11.9 & 8.5 & 0.1 & 0.05 & 0.003 & 0.004 & -3 & -1 & -2 & -3 \\
\hline 13 & 53 & 180 & 8 & $<2$ & 0.03 & 0.008 & 7.8 & 5.7 & 0.07 & 0.02 & 0.003 & 0.004 & -3 & -1 & -2 & -3 \\
\hline 14 & 167 & 265 & 8 & 3 & 0.19 & 0.14 & 1.6 & 0.9 & 0.09 & 0.05 & 0.004 & 0.01 & -3 & -2 & -2 & -3 \\
\hline 15 & 54 & 429 & 5 & 2 & 0.05 & 0.02 & 2.9 & 1.1 & 0.06 & 0.03 & 0.004 & 0.01 & -3 & -2 & -2 & -3 \\
\hline 16 & 21 & 329 & 4 & 7 & 0.1 & 0.5 & 0.2 & 0.2 & 0.01 & 0.01 & 0.009 & 0.02 & $\begin{array}{l}-2 \\
\end{array}$ & -3 & \begin{tabular}{|l|}
-3 \\
\end{tabular} & -3 \\
\hline 17 & 70 & 393 & 7 & 2 & 0.04 & 0.04 & 3.8 & 2.5 & 0.1 & 0.08 & 0.003 & 0.003 & -3 & -2 & -2 & -3 \\
\hline 18 & 101 & 203 & $<2$ & $<2$ & 0.03 & 0.03 & 6.0 & 4.5 & 0.1 & 0.08 & 0.003 & 0.003 & -4 & -2 & -1 & -3 \\
\hline 19 & 228 & 282 & 11 & 5 & 1.9 & 5 & 0.2 & $\begin{array}{ll}0.4 \\
\end{array}$ & 0.01 & 0.01 & \begin{tabular}{|l|}
0.01 \\
\end{tabular} & 0.02 & -2 & -3 & -3 & -3 \\
\hline 110 & 73 & 130 & 8 & 4 & 0.1 & 0.1 & 1.8 & 1.2 & 0.1 & 0.06 & 0.003 & 0.004 & -3 & -2 & -2 & -3 \\
\hline $\mid 111$ & 102 & 132 & $<2$ & $<2$ & 0.06 & 0.02 & 2.1 & 1.0 & 0.07 & 0.03 & 0.003 & 0.003 & -3 & -2 & -1 & -3 \\
\hline $\mid 12$ & 122 & 100 & $<2$ & $<2$ & 0.3 & $\begin{array}{l}1.1 \\
\end{array}$ & 2.0 & \begin{tabular}{|l|}
1.4 \\
\end{tabular} & 0.1 & 0.08 & 0.003 & 0.002 & $\begin{array}{l}-3 \\
\end{array}$ & -2 & $\begin{array}{l}-1 \\
\end{array}$ & -3 \\
\hline S1 & 13 & 1,798 & $<2$ & $<2$ & 0.01 & 0.006 & 24.6 & 11.0 & 0.03 & 0.01 & 0.003 & 0.003 & -3 & -2 & -2 & -3 \\
\hline S2 & 20 & 588 & $<2$ & $<2$ & 0.008 & 0.03 & 17.1 & 9.0 & 0.03 & 0.01 & 0.003 & 0.002 & $\begin{array}{l}-3 \\
\end{array}$ & -2 & -2 & -3 \\
\hline S3 & 31 & 948 & 6 & 24 & 0.002 & 0.002 & 0.03 & 0.01 & 0.01 & 0.003 & \begin{tabular}{|l|}
0.02 \\
\end{tabular} & 0.01 & -4 & -5 & -3 & -3 \\
\hline S4 & 62 & 78 & 13 & 7 & 0.005 & 0.02 & 1.5 & 0.7 & 0.01 & 0.01 & 0.004 & 0.004 & $\begin{array}{l}-4 \\
\end{array}$ & -2 & $\begin{array}{l}-3 \\
\end{array}$ & -3 \\
\hline S5 & 41 & 43 & 4 & 15 & 0.03 & 0.02 & 0.04 & 0.01 & 0.05 & 0.02 & 0.003 & 0.002 & -3 & -3 & -2 & -4 \\
\hline S6 & 47 & 53 & 4 & 4 & 0.02 & 0.01 & 0.04 & 0.01 & 0.05 & 0.04 & 0.004 & 0.003 & $\begin{array}{l}-3 \\
\end{array}$ & -3 & -2 & -3 \\
\hline S7 & 67 & 306 & 12 & 16 & 0.004 & 0.003 & 0.02 & 0.01 & 0.02 & 0.02 & \begin{tabular}{|l|}
0.01 \\
\end{tabular} & 0.01 & -4 & -4 & -3 & -3 \\
\hline S8 & 36 & 132 & 6 & 21 & 0.004 & 0.002 & 0.3 & $\begin{array}{l}0.2 \\
\end{array}$ & 0.02 & 0.01 & 0.01 & 0.01 & $\begin{array}{l}-4 \\
\end{array}$ & -3 & -2 & -4 \\
\hline S9 & 77 & 68 & 29 & 20 & 0.002 & 0.001 & 0.01 & 0.01 & 0.01 & 0.01 & 0.01 & 0.01 & -5 & -4 & -4 & -3 \\
\hline $\mathrm{S} 10$ & 1,050 & 156 & 137 & 13 & 0.007 & 0.005 & 0.02 & 0.01 & 0.02 & 0.01 & 0.04 & 0.04 & -5 & -4 & -4 & -3 \\
\hline $\mathrm{S} 11$ & 46 & 116 & 4 & 5 & 0.003 & 0.002 & 0.01 & 0.005 & 0.02 & 0.02 & 0.01 & 0.02 & -4 & -4 & -2 & -3 \\
\hline $\mathrm{H} 1$ & 36 & 2 & 2 & 2 & 0.003 & 0.005 & 0.01 & 0.01 & 0.01 & 0.01 & 0.002 & 0.000 & $\begin{array}{l}-4 \\
\end{array}$ & -2 & $\begin{array}{l}-2 \\
\end{array}$ & -3 \\
\hline $\mathrm{H} 2$ & 31 & 4 & 4 & 3 & 0.003 & 0.005 & 0.01 & 0.03 & 0.01 & 0.004 & 0.003 & 0.005 & -4 & -2 & -3 & -3 \\
\hline $\mathrm{H} 3$ & 190 & 2 & 14 & 2 & 0.004 & 0.003 & 0.08 & 0.05 & 0.01 & 0.004 & 0.002 & 0.000 & $\begin{array}{l}-5 \\
\end{array}$ & -1 & \begin{tabular}{|l|} 
\\
\end{tabular} & -3 \\
\hline $\mathrm{H} 4$ & 25 & 44 & 2 & 2 & 0.002 & 0.001 & 0.03 & 0.01 & 0.01 & 0.005 & 0.005 & 0.007 & -4 & -3 & -2 & -3 \\
\hline $\mathrm{H} 5$ & 23 & 6 & 6 & 2 & 0.009 & 0.004 & 0.02 & 0.005 & 0.1 & 0.03 & 0.003 & 0.003 & -3 & -2 & -2 & -3 \\
\hline $\mathrm{H} 6$ & 36 & 100 & 2 & 2 & 0.005 & 0.004 & 0.02 & 0.01 & 0.01 & 0.01 & 0.006 & 0.005 & -4 & -4 & -2 & -2 \\
\hline $\mathrm{H} 7$ & 46 & 3 & 2 & 2 & 0.1 & 0.2 & 0.01 & 0.004 & 0.01 & 0.01 & 0.003 & 0.003 & -3 & -3 & -2 & -3 \\
\hline $\mathrm{H} 8$ & 130 & 10 & 2 & 2 & 0.2 & 1.4 & 0.03 & \begin{tabular}{|l|}
0.01 \\
\end{tabular} & 0.04 & 0.1 & 0.003 & 0.002 & -3 & -3 & -2 & -3 \\
\hline $\mathrm{H} 9$ & 84 & 5 & 3 & 2 & 0.01 & 0.01 & 0.02 & 0.01 & 0.02 & 0.02 & 0.004 & 0.004 & -4 & -2 & -2 & -3 \\
\hline $\mathrm{H} 10$ & 102 & 2 & 5 & 2 & 0.04 & 0.03 & 0.02 & 0.01 & 0.2 & 0.1 & 0.003 & 0.003 & -3 & -2 & -1 & -3 \\
\hline $\mathrm{H} 11$ & 127 & 2 & 14 & 2 & 6.9 & 3.8 & 0.1 & 0.5 & 0.5 & 0.3 & 0.003 & 0.003 & $\begin{array}{l}-1 \\
\end{array}$ & -1 & $\begin{array}{l}-1 \\
\end{array}$ & -3 \\
\hline $\mathrm{H} 12$ & 251 & 2 & 2 & 2 & 0.3 & 1.3 & 0.02 & 0.01 & 0.03 & 0.10 & 0.002 & 0.002 & -3 & -2 & -2 & -3 \\
\hline $\mathrm{H} 13$ & 116 & 2 & 2 & 2 & 0.008 & 0.007 & 0.01 & 0.01 & 0.01 & 0.01 & 0.003 & 0.002 & -4 & -2 & -2 & -3 \\
\hline $\mathrm{H} 14$ & 74 & 289 & 13 & 2 & 5 & 4 & 0.02 & 0.01 & 0.3 & 0.3 & 0.002 & 0.002 & -1 & -4 & -2 & -3 \\
\hline M1 & 68 & 18 & 7 & 2 & 0.01 & 0.004 & 0.1 & \begin{tabular}{|l|}
0.1 \\
\end{tabular} & 0.01 & 0.01 & 0.006 & 0.006 & -4 & -2 & -3 & -3 \\
\hline $\mathrm{M} 2$ & 35 & 10 & 2 & 2 & 0.4 & 0.7 & 0.1 & 0.2 & 0.06 & 0.04 & 0.004 & 0.004 & -2 & -2 & -2 & -3 \\
\hline M3 & 15 & 4 & 2 & 2 & 0.001 & 0.001 & 0.02 & 0.01 & 0.01 & 0.005 & 0.002 & 0.000 & -4 & -2 & -2 & -3 \\
\hline M4 & 92 & 2 & 2 & 2 & 1.7 & 1.6 & 0.02 & 0.02 & 0.03 & 0.03 & 0.003 & 0.002 & -2 & -2 & -2 & -3 \\
\hline M5 & 194 & 11 & 13 & 2 & 0.007 & 0.003 & 0.03 & \begin{tabular}{|l|}
0.01 \\
\end{tabular} & 0.01 & 0.004 & 0.003 & 0.002 & $\begin{array}{l}-4 \\
\end{array}$ & -3 & \begin{tabular}{|l|}
-3 \\
\end{tabular} & -3 \\
\hline M6 & 74 & 2 & 11 & 2 & 0.02 & 0.008 & 0.03 & 0.01 & 0.05 & 0.01 & 0.003 & 0.002 & -3 & -2 & -2 & -3 \\
\hline M7 & 73 & 104 & 20 & 3 & 0.03 & 0.009 & 0.06 & 0.03 & 0.15 & 0.06 & 0.003 & 0.002 & $\begin{array}{l}-3 \\
\end{array}$ & -3 & -2 & -3 \\
\hline M8 & 34 & 63 & 4 & 6 & 0.01 & 0.004 & 0.03 & 0.01 & 0.05 & 0.02 & 0.005 & 0.003 & -3 & -3 & -2 & -3 \\
\hline M9 & 102 & 3 & 2 & 2 & 0.01 & 0.01 & 0.02 & 0.005 & 0.01 & 0.01 & 0.006 & 0.004 & $\begin{array}{l}-4 \\
\end{array}$ & -2 & \begin{tabular}{|l|}
-2 \\
\end{tabular} & -3 \\
\hline M10 & 53 & 5 & 2 & 2 & 0.004 & 0.003 & 0.02 & 0.004 & 0.02 & 0.01 & 0.004 & 0.003 & -4 & -2 & -2 & -3 \\
\hline M11 & 16 & 10 & 2 & 2 & 0.004 & 0.004 & 0.01 & 0.01 & 0.06 & 0.03 & 0.003 & 0.003 & $\begin{array}{l}-4 \\
\end{array}$ & -3 & -2 & -3 \\
\hline M12 & 16 & 2 & 3 & 2 & 0.008 & 0.05 & 0.01 & 0.003 & 0.06 & 0.03 & 0.003 & 0.003 & -3 & -2 & -2 & -3 \\
\hline
\end{tabular}


Figure S1. Particle size distributions of the unweathered waste rock used in the field barrel kinetic experiments.
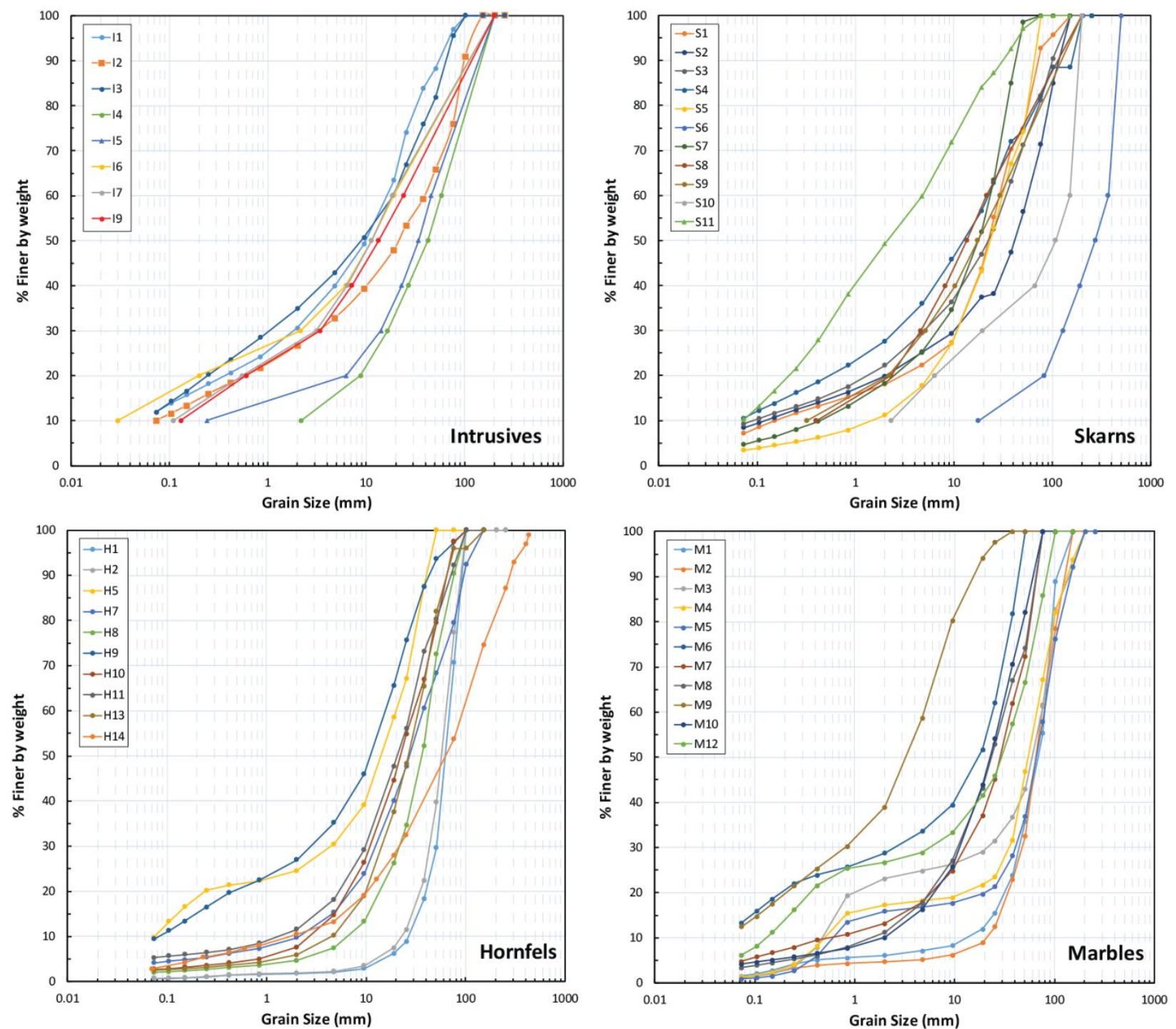
Figure S2. Long-term evolution of drainage concentrations of $\mathrm{Cu}, \mathrm{Fe}$ and $\mathrm{Zn}$ from the field-barrel kinetic tests with various types of waste rock. Drainage samples from the 49 field-barrel experiments are here aggregated per waste-rock type (intrusive, skarn, hornfels, marble). Note the logarithmic y-axes.
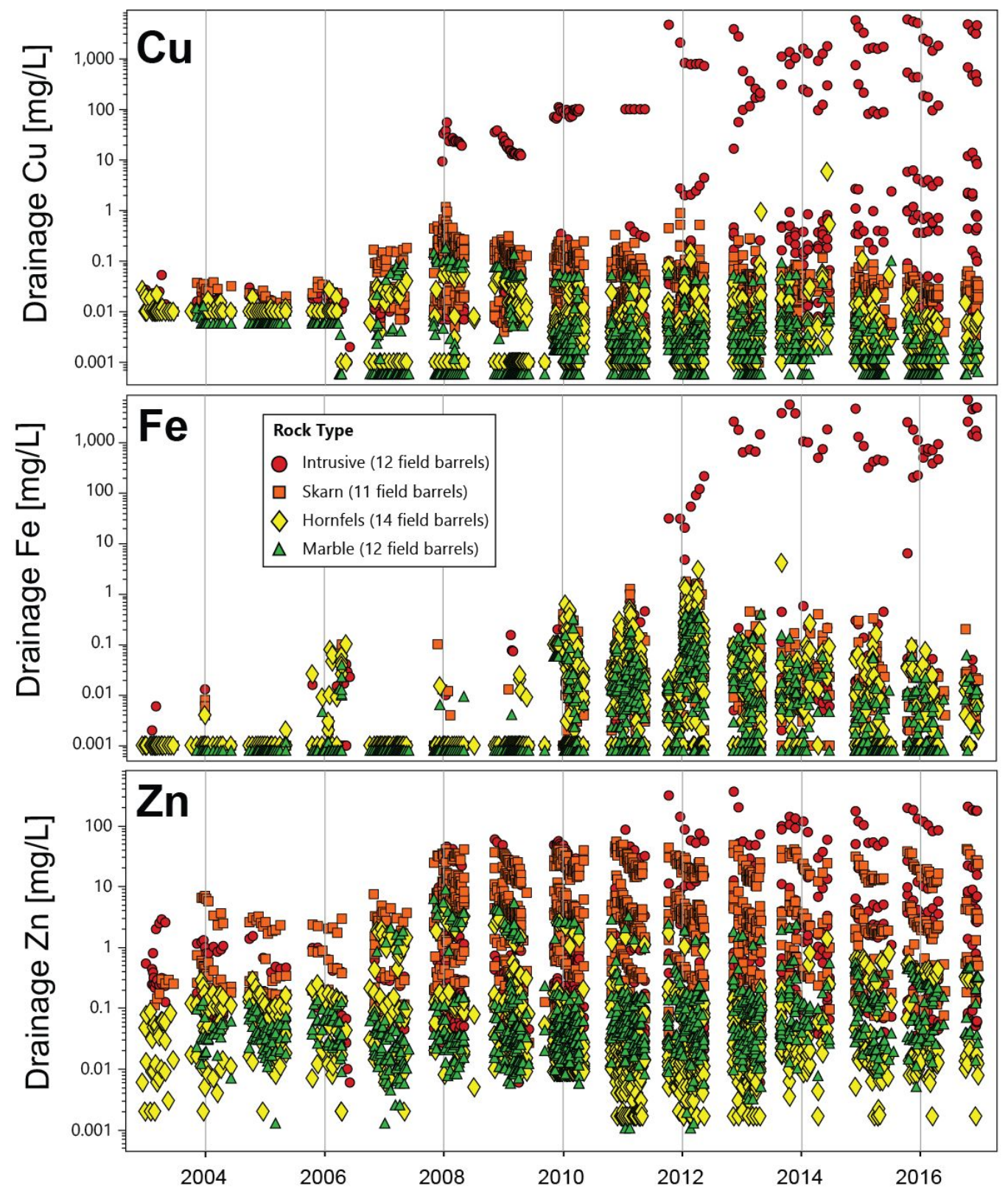
Figure S3. Long-term evolution of drainage concentrations of $\mathrm{V}, \mathrm{W}, \mathrm{Cr}$ and $\mathrm{B}$ from the field-barrel kinetic tests with various types of waste rock. Drainage samples from the 49 field-barrel experiments are here aggregated per waste-rock type (intrusive, skarn, hornfels, marble). Note the logarithmic y-axes.
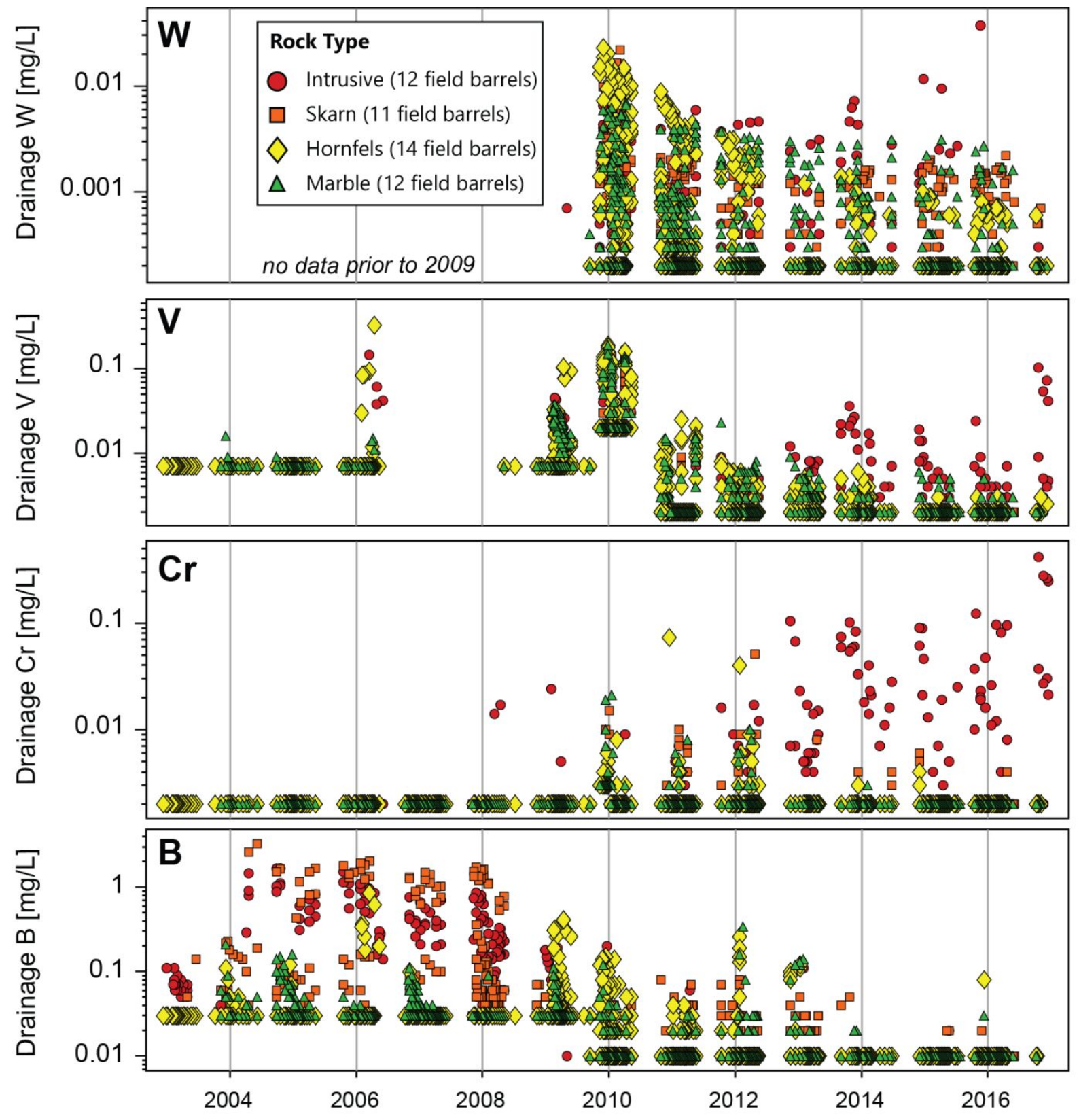
Figure S4. Bivariate correlation plots between a selection of elements measured in the drainage from the field-barrel kinetic tests (not aggregated by waste-rock lithology). While strong correlations sporadically exist for individual field barrels, generalization across similar lithology generally obscures these correlations. An exception is the correlation between $\mathrm{Ca}$ and sulfate (top right), which is caused by a dominant solubility control of secondary gypsum on their concentrations in drainage from most field barrels.
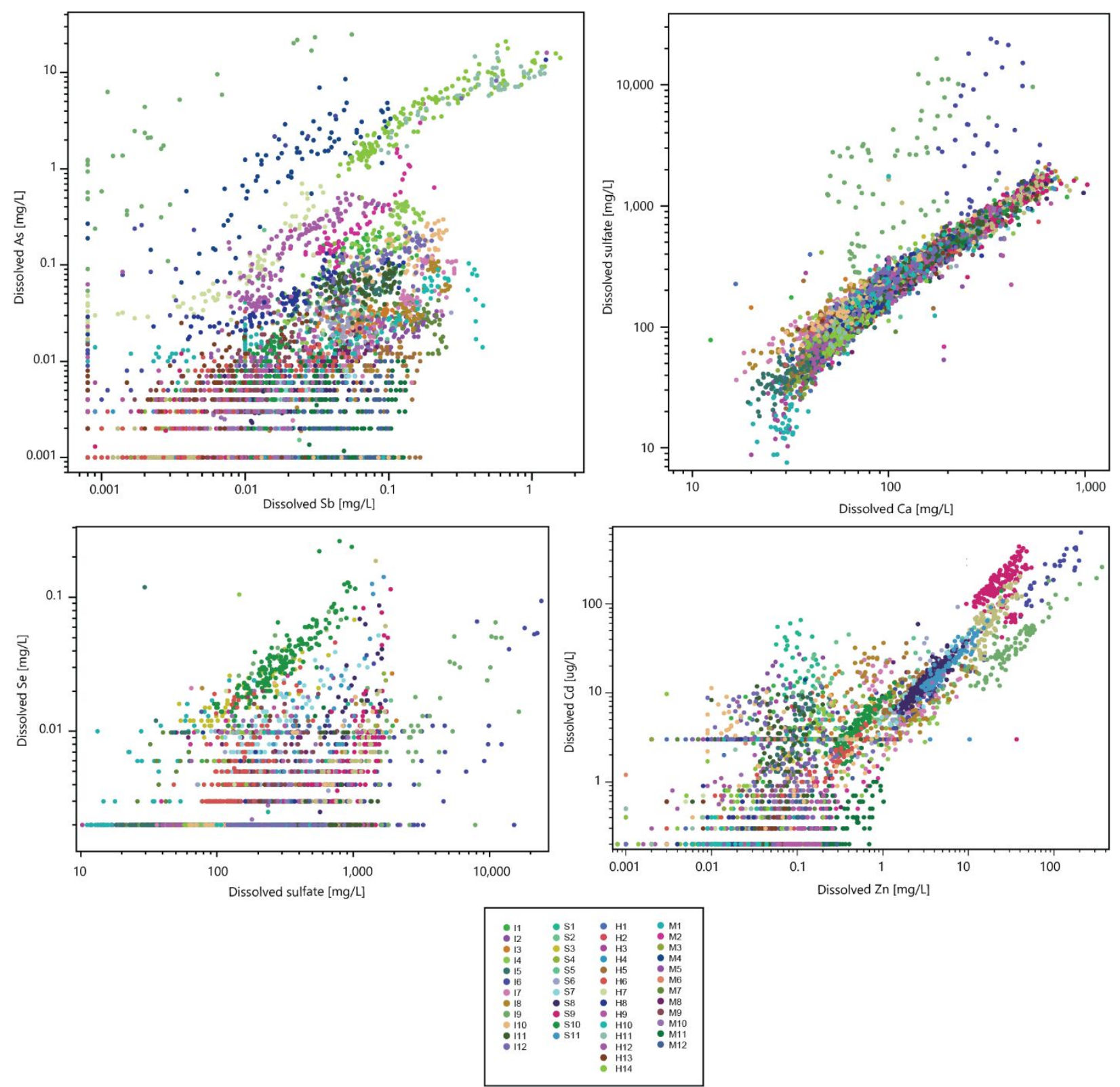
Figure S5. SEM-BSE images and semi-quantitative elemental analysis of EDS spectra from spot locations in three skarn waste rock grains from field barrel S10. The EDS spectra were used to determine the wt- $\%$ of elements in primary minerals and secondary phases mineral replacements and alteration rims.

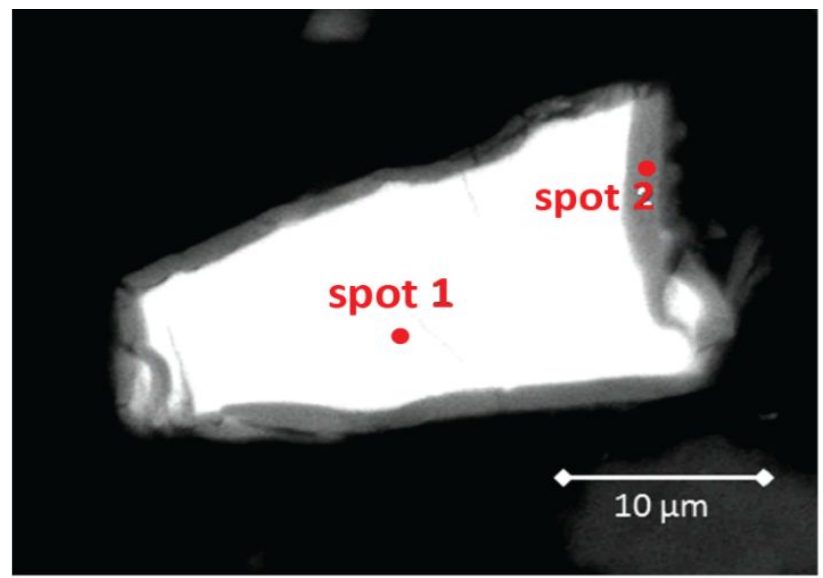

\begin{tabular}{|c|c|c|}
\hline & Spot 1 & Spot 2 \\
\hline $\mathrm{Al}$ & 0.1 & 1.9 \\
$\mathrm{As}$ & $<0.1$ & 0.6 \\
$\mathrm{Ca}$ & 0.1 & 1.7 \\
$\mathrm{Cu}$ & 35 & 19 \\
$\mathrm{Fe}$ & 33 & 30 \\
$\mathrm{~K}$ & $<0.1$ & 0.4 \\
$\mathrm{Mg}$ & $<0.1$ & 1.1 \\
$\mathrm{O}$ & 0.7 & 25 \\
$\mathrm{~S}$ & 31 & 11 \\
$\mathrm{Si}$ & 0.1 & 7.3 \\
$\mathrm{Zn}$ & 0 & 2.0 \\
\hline Total: & 100 & 100 \\
\hline
\end{tabular}

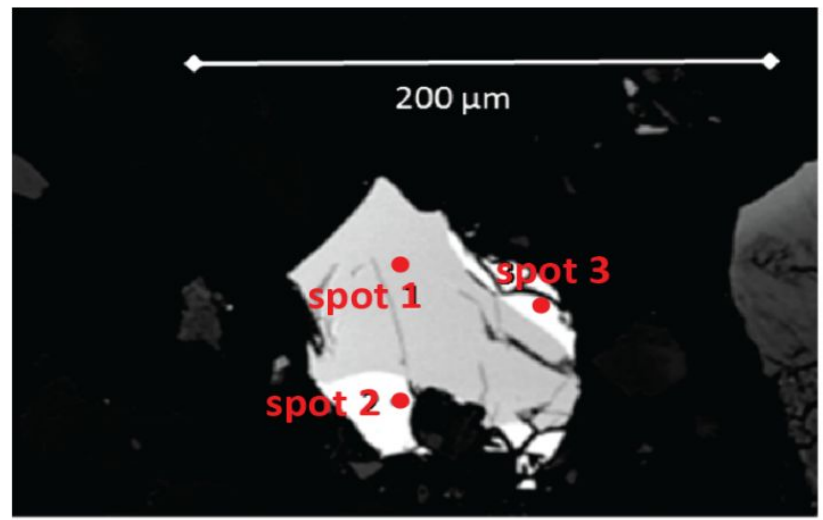

\begin{tabular}{|c|c|c|c|}
\hline & Spot 1 & Spot 2 & Spot 3 \\
\hline $\mathrm{As}$ & $<0.1$ & 10 & 7 \\
$\mathrm{C}$ & $<0.1$ & 47 & 56 \\
$\mathrm{Ca}$ & 19 & 0.2 & 0.2 \\
$\mathrm{Cu}$ & 29 & 20 & 16 \\
$\mathrm{Fe}$ & 26 & 2 & 2 \\
$\mathrm{O}$ & 1 & 8 & 8 \\
$\mathrm{~S}$ & 26 & 10 & 7 \\
$\mathrm{Sb}$ & $<0.1$ & 0.6 & 0.7 \\
$\mathrm{Si}$ & $<0.1$ & 0.4 & 0.1 \\
$\mathrm{Zn}$ & $<0.1$ & 2 & 2 \\
\hline Total: & 100 & 100 & 99.8 \\
\hline
\end{tabular}

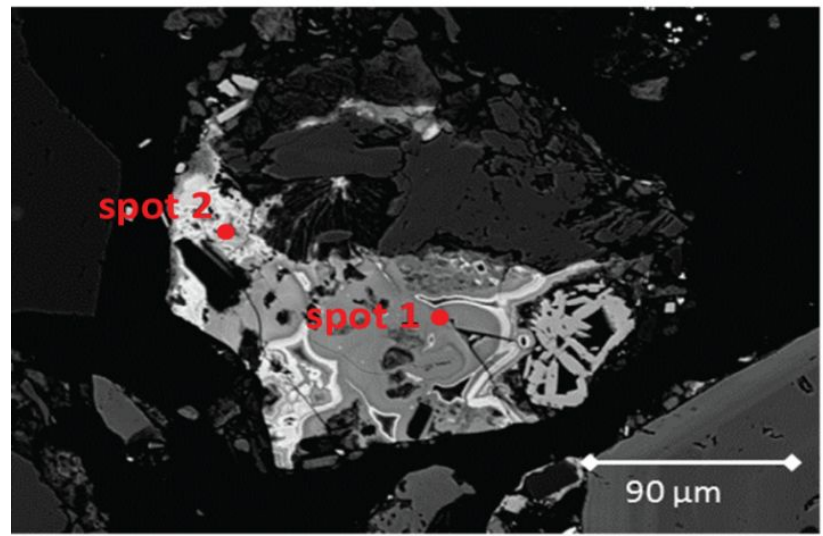

\begin{tabular}{|c|c|c|}
\hline & Spot 1 & Spot 2 \\
\hline $\mathrm{S}$ & $<0.1$ & 3 \\
$\mathrm{Fe}$ & 1 & 2 \\
$\mathrm{O}$ & 26 & 21 \\
$\mathrm{Si}$ & 12 & 3 \\
$\mathrm{Cu}$ & 18 & 20 \\
$\mathrm{Zn}$ & 13 & 6 \\
$\mathrm{Mn}$ & 4 & 21 \\
$\mathrm{As}$ & $<0.1$ & 7 \\
$\mathrm{C}$ & 23 & 16 \\
$\mathrm{Ca}$ & 0.3 & 1 \\
$\mathrm{Al}$ & 1 & $<0.1$ \\
$\mathrm{Mg}$ & 1 & $<0.1$ \\
\hline Total: & 100 & 99 \\
\hline
\end{tabular}


Figure S6. Bivariate correlation plots between molybdenum and sulfate in the drainage from the field-barrel kinetic tests (not aggregated by waste-rock lithology).

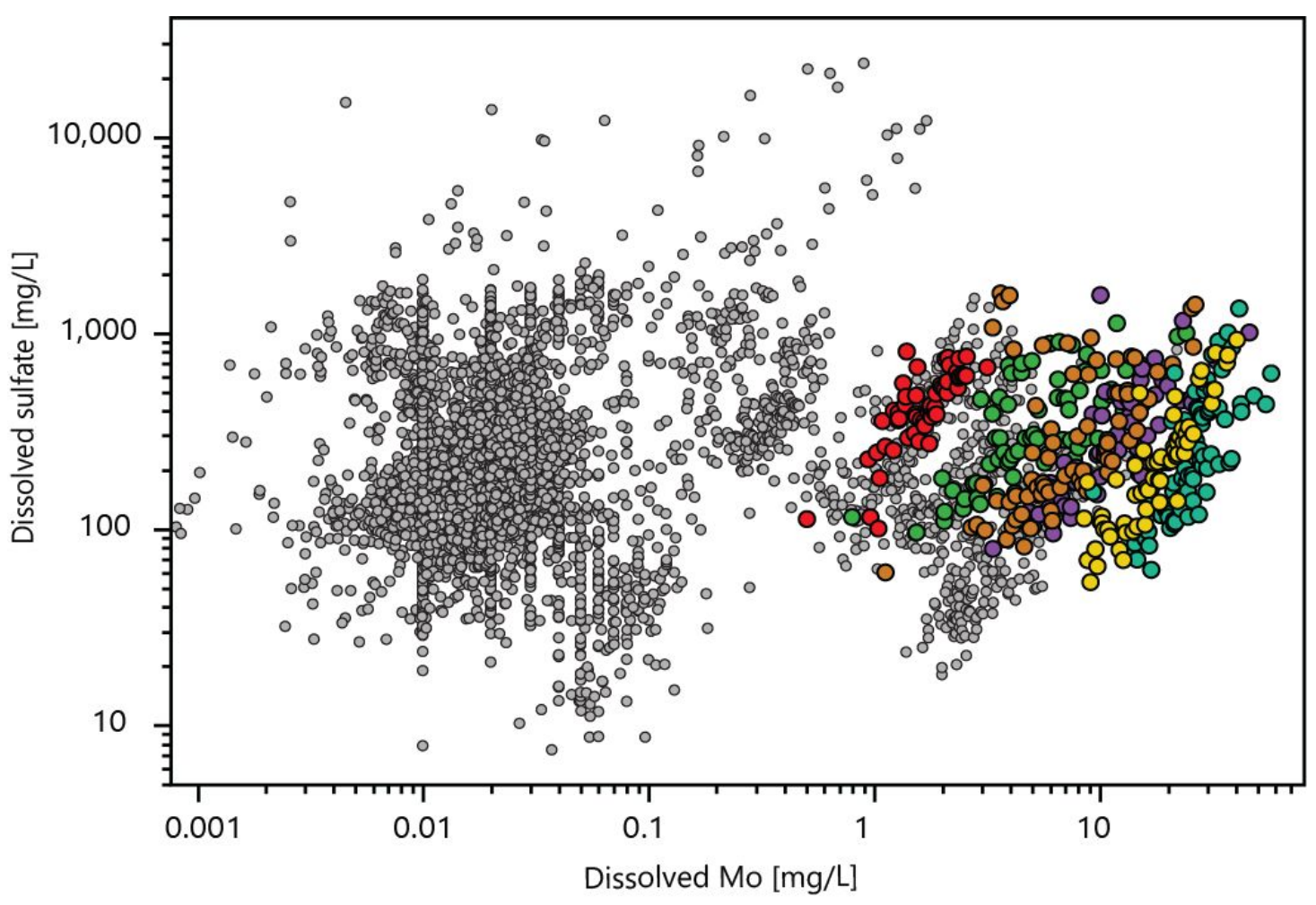

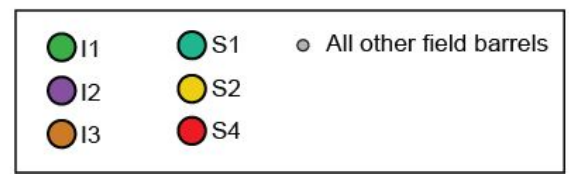


Figure S7. Correlations between the $\mathrm{Se}$ and $\mathrm{S}$ concentrations in waste rock at Antamina (top; linear axes) and in the drainages of a selection of field barrels with either skarn or intrusive waste rock (bottom; double-logarithmic axes). The dotted black lines indicate linear-regression trend lines (intrusive and skarn waste rock only) for which the corresponding coefficients of determination $\left(R^{2}\right)$ are given.
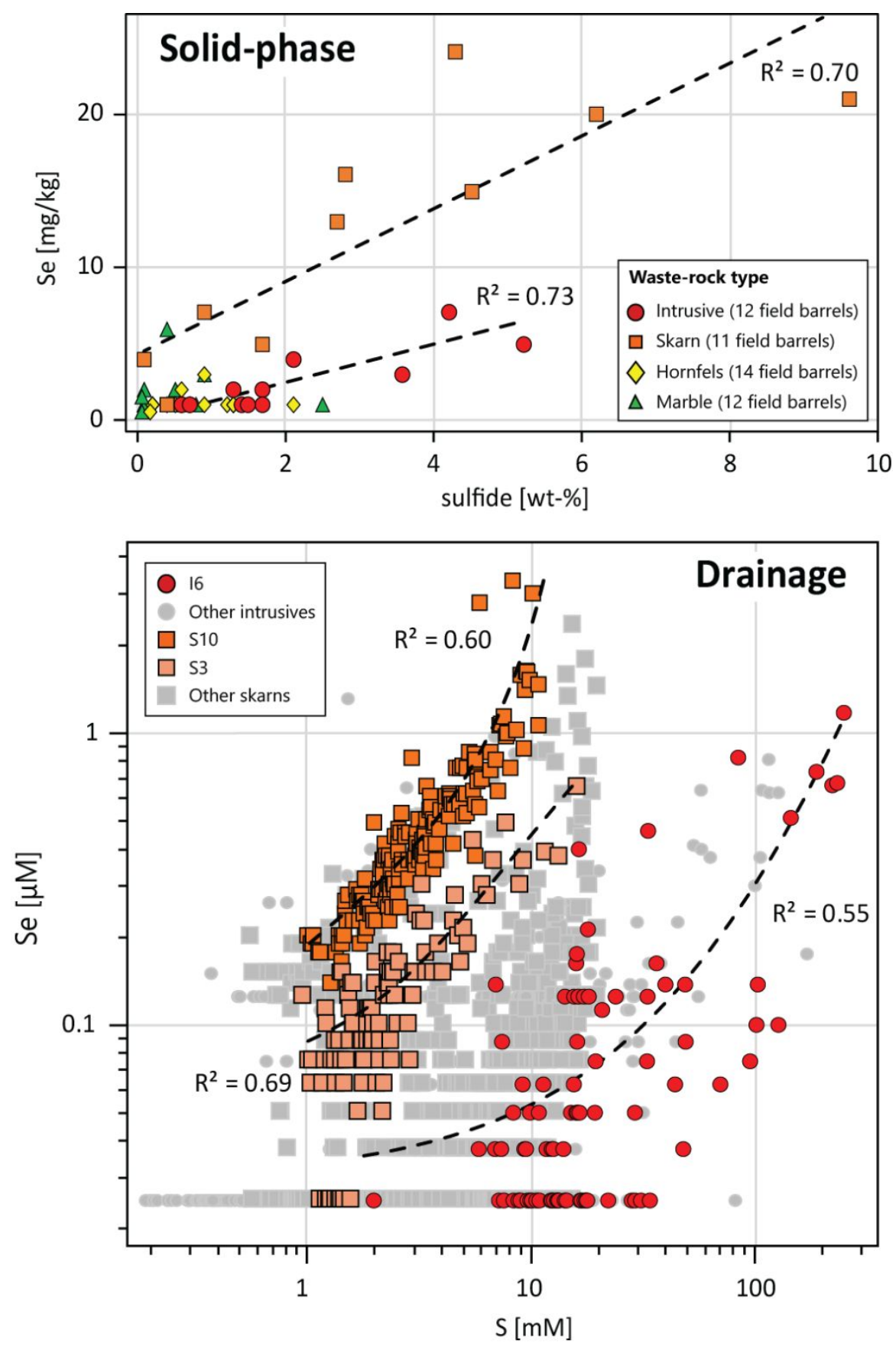
Figure S8. Correlation plots between dissolved sulfate concentrations and $\mathrm{pH}$ in the drainage from all 49 field-barrel kinetic tests. Aqueous drainage chemistry measurements are here aggregated per waste-rock type. Logarithmic y-axis.

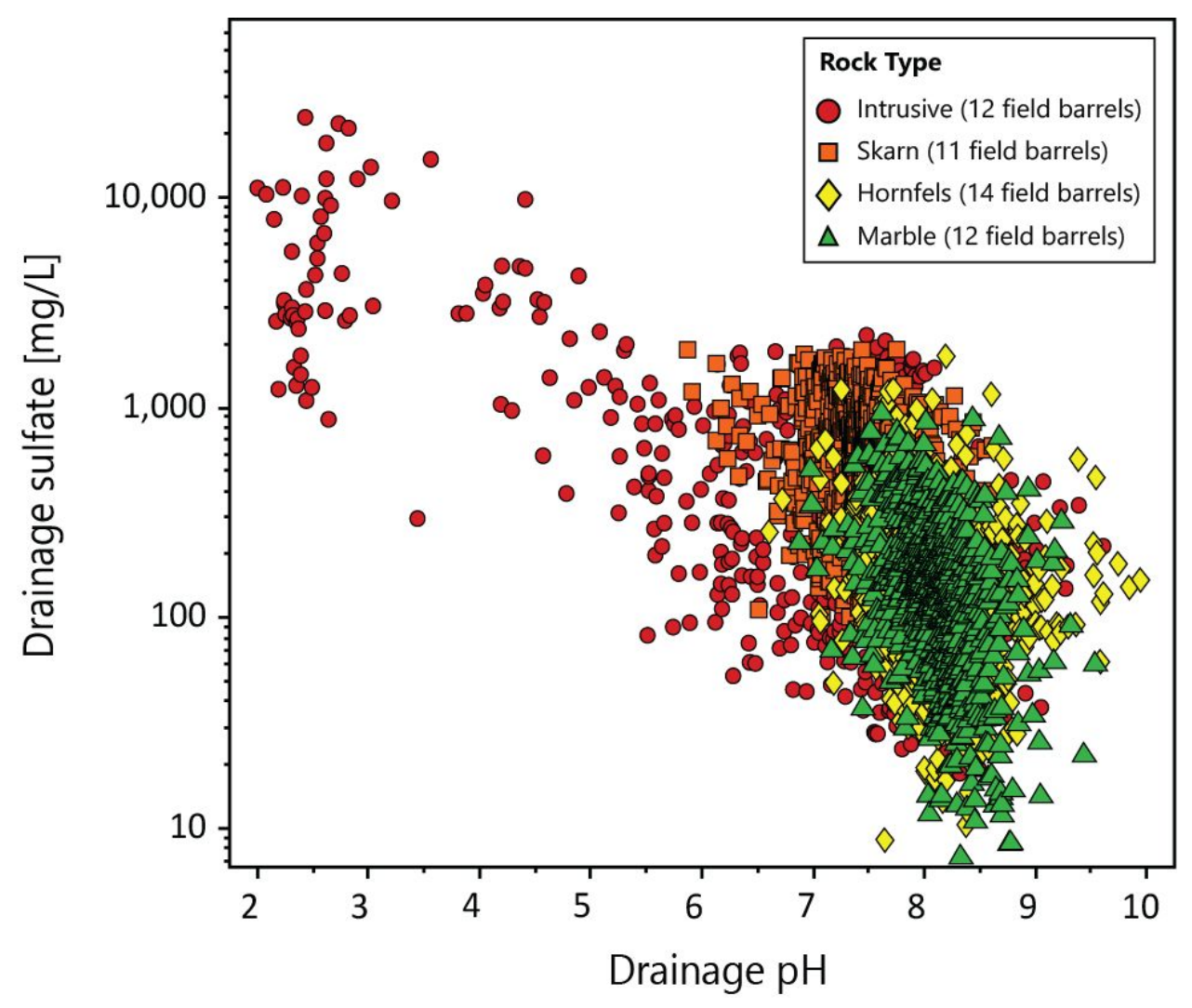


Figure S9. Long-term evolution of drainage concentrations of $\mathrm{Fe}, \mathrm{As}, \mathrm{Se}, \mathrm{Mo}$, and $\mathrm{Sb}$ and drainage $\mathrm{pH}$ from field barrel 19 , showing the drainage acidification and concurrent oxyanion release upon dissolution of secondary Fe minerals. Note the logarithmic yaxes.
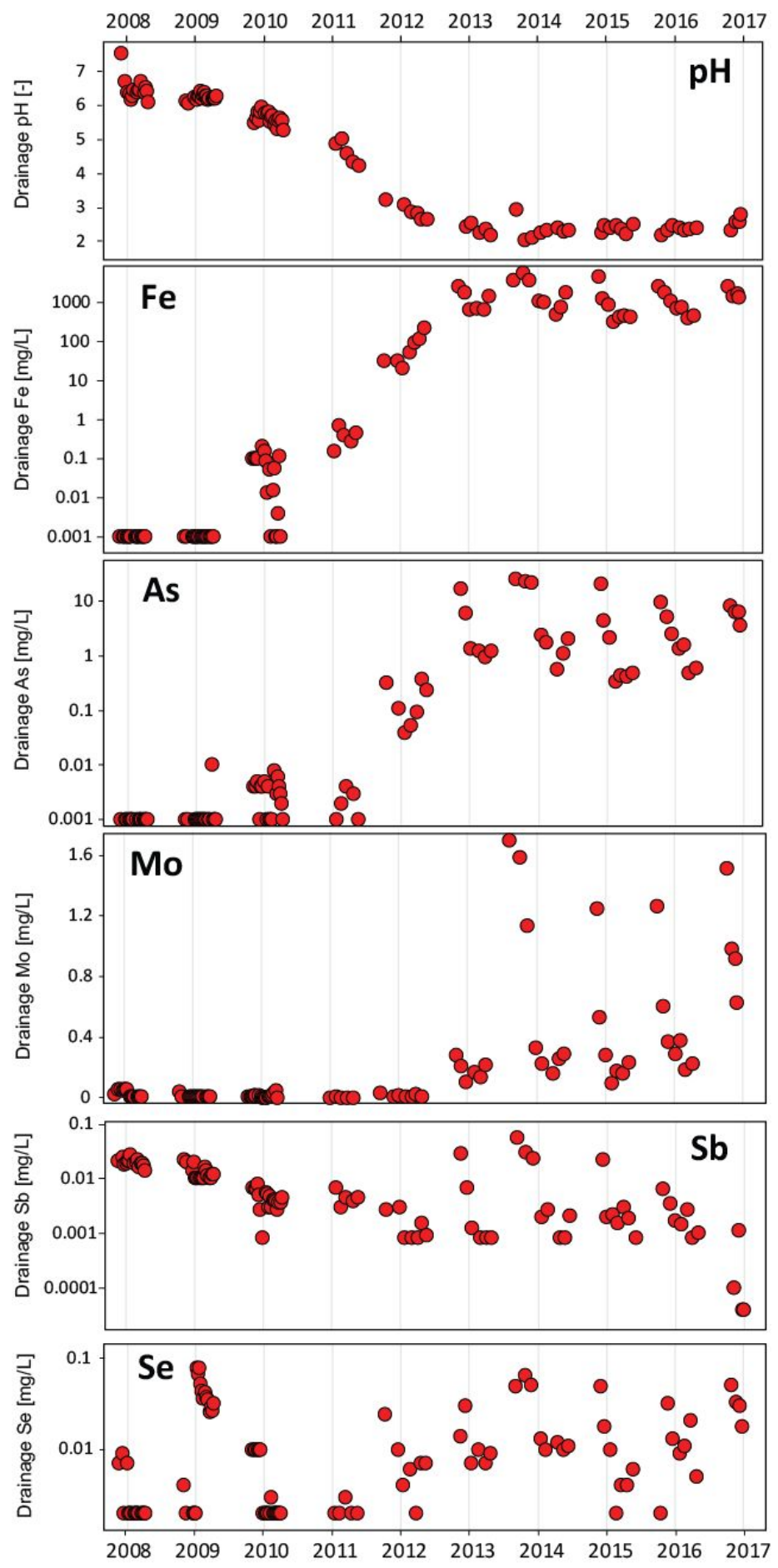
Figure S10. Simulation of the sorption behavior of As, Mo and Se onto hydrous ferric oxide (HFO) under variable drainage chemistries as detailed in Table S4, while accounting for competitive surface complexation and variable aqueous speciation, performed using PHREEQC and the MINTEQ.v4 database. The plots illustrate the fraction of HFO-sorbed oxyanions over their total concentration (mol-\%). The dominant surface complexes for the oxyanions are indicated for each approximate $\mathrm{pH}$ domain; the grey shading corresponds to the $\mathrm{pH}$ regions where oxyanions are mobile as observed from the field data and discussed in the text.
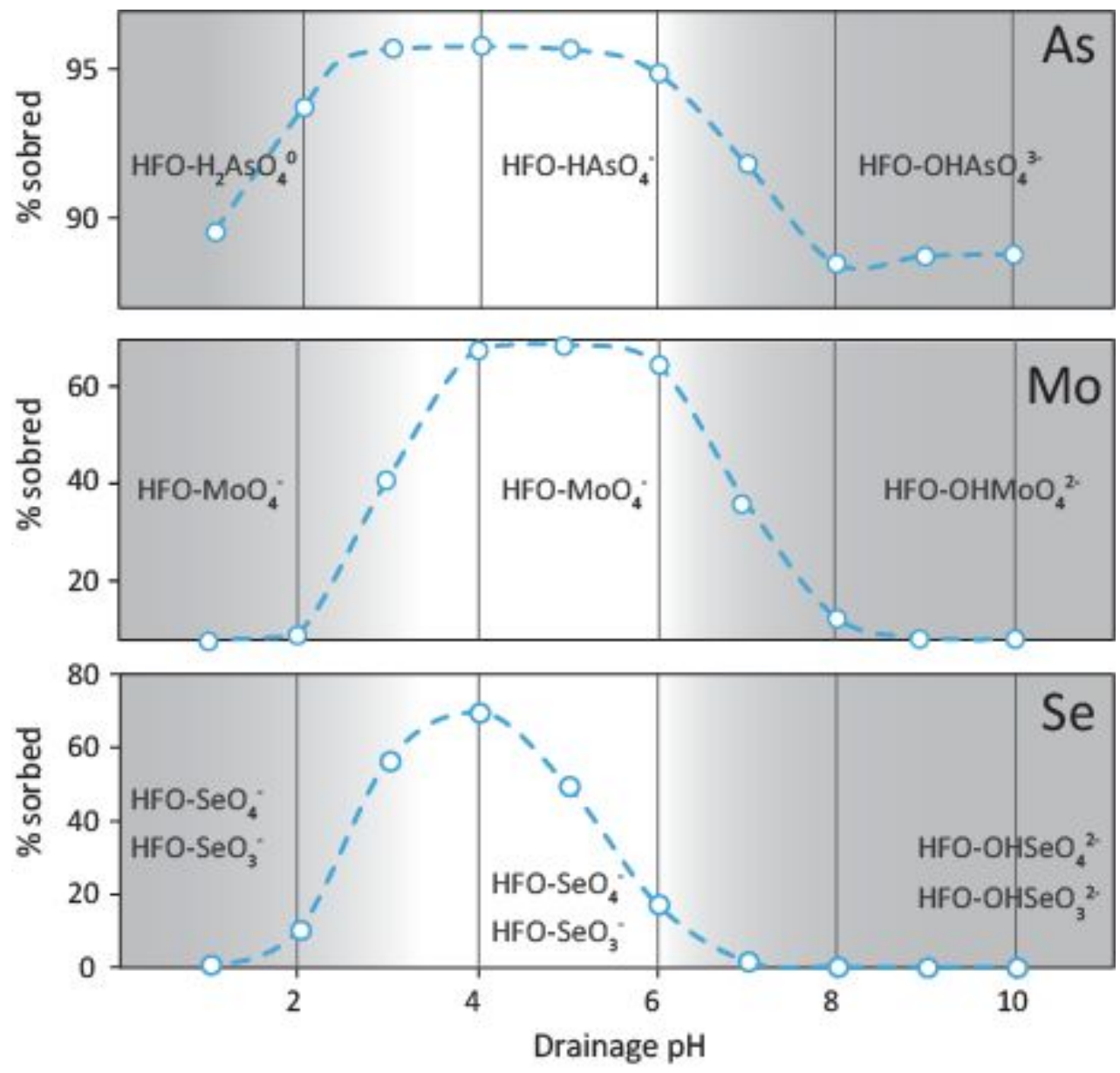